\title{
La gimnasia como práctica en la Educación Primaria. Recorridos, avances y revisiones
}

\author{
Maria Emilia Napolitano \\ emilianapolitano05@gmail.com
}

\begin{abstract}
Resumen:
La gimnasia como práctica social, política, histórica, educativa y cultural ha ido evolucionando a lo largo de años desde la implementación de la Ley $N^{\circ} 1420$ hasta la actualidad como parte de las propuestas de Educación Física del Diseño Curricular de la Provincia de Buenos Aires. Ha transitado por varios procesos que la fueron marcando y le han dado identidad: gimnasia militarizada, ejercicios físicos, gimnasia como contenido de la Educación Física, gimnasia como práctica repetitiva de las técnicas y sus métodos y, en una última corriente o paradigma, la gimnasia como construcción del saber. En esta pesquisa el objeto de estudio se centra en el seguimiento de cinco momentos de la gimnasia que han marcado los lineamientos de la educación a través del análisis, la indagación y la reflexión de bibliografía pertinente en torno a la temática. Cada uno de estos momentos, han permitido comprender cómo la gimnasia se ha configurado con los años y qué sentido tuvo (y tiene) en el sistema educativo. (Re)pensar, (re)valorizar y analizarla permiten la apertura a una práctica considerada realmente como contenido de la Educación Física. Una gimnasia que se vuelva a enseñar, pero con otras herramientas y otras perspectivas.
\end{abstract}

Palabras clave: Gimnasia, Práctica, Sistema educativo, Diseño curricular.

\section{ABstraCt:}

Gymnastics as a social, political, historical, educational and cultural practice has evolved over the years from the implementation of Law No. 1420 to the present as part of the proposals of Physical Education of Curriculum Design of the Province of Buenos Aires. He has gone through several processes that have marked him and given him identity: militarized gymnastics, physical exercises, gymnastics as a content of Physical Education, gymnastics as a repetitive practice of techniques and methods and, in a last current or paradigm, the gymnastics as construction of knowledge. In this research, the object of study focuses on the follow-up of five moments of gymnastics that have marked the guidelines of education through the analysis, inquiry and reflection of pertinent bibliography on the subject. Each of these moments, have allowed to understand how gymnastics has been configured over the years and what sense it had (and has) in the educational system. (Re) thinking, (re) valuing and analyzing it allow the opening to a gym considered really as content of Physical Education. A gymnastics that is re-taught, but with other tools and other perspectives. KeYworDs: Gymnastics, Practice, Educational system, Curricular Design.

\section{INTRODUCCIÓN}

La constitución de la gimnasia como práctica política, educativa, histórica, social y económica se ha ido modificando a lo largo de los años. Estas modificaciones causaron la transformación de sus propios métodos de enseñanza, la configuración de varias corrientes de la gimnasia con diversos objetivos, el dictado de las mismas y las controversias que acaecieron entre instructores, maestros y profesores en Educación Física, el entrenamiento de las diferentes capacidades coordinativas y condicionales de acuerdo a la corriente de gimnasia, las planificaciones de los docentes y la desaparición de la gimnasia en la misma, el interés y/o desinterés de los alumnos y alumnas al practicarla, los saberes previos, los pocos cursos de capacitación, etc.

En esta pesquisa la centralidad se verá reflejada en distintos momentos que han atravesado a la gimnasia: 1) los ejercicios físicos se oficializan con la Ley $\left.\mathrm{N}^{\circ} 1420,2\right)$ la gimnasia y configuración de la Educación Física en nuestro país junto a Romero Brest, 3) la gimnasia utilizada y atravesada por los paradigmas de la Educación Física: el paradigma físico deportivo y el psicomotor, 4) la gimnasia de los Diseños Curriculares 
en el período de 2007 y 2017, y 5) la nueva curricula educativa en el 2018 y el rol de la gimnasia. Cada uno de estos recortes históricos permitieron configurar diversas prácticas de la gimnasia. Algunas poco pedagógicas, otras por imitación, la mayoría con el único objeto de entrenar lo corporal, algunas planificadas con poco entusiasmo, etc.

La gimnasia necesita ser pensada, planeada y construirla de un modo diferente. A lo largo de la investigación se analizará algunos recortes seleccionados para la misma y se propondrá un modo de ser distinto de las prácticas de la gimnasia.

\section{Primer momento. Los ejercicios físicos SE Oficializan con LA Ley No 1420. Los primeros pasos de la gimnasia en el Sistema Educativo}

La gimnasia en el marco del sistema educativo aparece con la Ley $\mathrm{N}^{\circ} 1420$ en 1884 quien prescribe la educación básica, obligatoria y neutralmente religiosa. Con el ingreso de esta Ley los saberes se unifican de acuerdo a las disciplinas, ocupándose de regularizar el cuerpo a través de los ejercicios físicos, la gimnasia y los ejercicios militares (saberes prácticos que atravesaban la curricula escolar).

Estos saberes como parte de los contenidos mínimos de la instrucción básica proponían para las mujeres el conocimiento de labores de manos, nociones de economía doméstica, puericultura, actividades rítmicas, gimnasia femenina, danzas y ciertos juegos. Para los hombres el destino era distinto se les enseñaba nociones de agricultura, ganadería, instrucción cívica. Aunque a partir de cuarto grado se les enseñaban ejercicios militares como la marcha, la carrera, los movimientos unificados de flanco, media vuelta, alineaciones, posiciones fundamentales, conversiones, formación, los ejercicios se realizan al ritmo de tambor. y, como si no fuera poco, se comenzó a armar a los niños con palos para luego convertirlos en fusiles en las propias escuelas.

Como es evidente, esta gimnasia que se proponía, tenía un carácter militar poco pedagógico y atravesado por cuestiones de género que tenía por objetivo favorecer y dirigir el desarrollo moral, intelectual y físico de todo niño de seis a catorce años de edad. Es entendible esta perspectiva, ya que el cuerpo para la época era considerado como un organismo conformado por huesos, articulaciones, músculos y sistemas, y por ello había que entrenarlo. En este sentido, se entendía que era necesario ejercer una acción comprometida sobre el sistema nervioso, la coordinación, los movimientos, lo físico, lo fisiológico y lo psicológico.

Con la Ley 1420, la gimnasia o gimnástica quedó relegada a las prácticas militares. Prácticas metódicas, directivas, sin preguntas, ni respuestas, que imponían el orden, hablaban de disciplina (y atravesaban el cuerpo), con un vocabulario adecuado de los movimientos para que los alumnos no lo modifiquen (todos debían hacer exactamente el mismo movimiento con una técnica rigurosa) y, por ejemplo, con movimientos lentos para aprenderlos mejor. Pablo Scharagrodsky en sus investigaciones sobre este tipo de gimnasia describía cómo eran regularizados los movimientos: "el alumno varón debe hallarse bien cuadrado a su frente, observando para ello los principios que siguen: talones en una misma línea y unidos, siempre que lo permita la configuración del niño. Punta de los pies, vueltas hacia afuera, formando con ellas un ángulo algo menor que el recto. Las piernas tendidas sin hacer fuerza en las rodillas. El peso del cuerpo sobre las caderas, y el pecho un poco inclinado adelante, cuidando no sacar el vientre. Los hombros algo retirados y a la misma altura. Los brazos naturalmente caídos, sin unir los codos al cuerpo; las manos entreabiertas, con los pulgares rozando la costura del pantalón. La cabeza derecha con naturalidad, la barbilla un poco recogida y a la vista al frente" (Scharagrodsky, 2006, p.115).

Este tipo de gimnasia regularizaba las prácticas, los cuerpos y los discursos en el Sistema Educativo; un sistema específico que se estaba conformando de acuerdo a los saberes culturales de la época. En este contexto, los niños eran poco a poco preparados para la guerra, y las niñas para ser futuras mujeres de bien que sirvan a sus futuros hombres. 


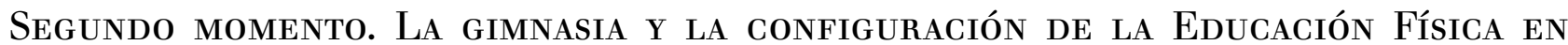 NUESTro PAÍ́s JUNTo Romero Brest}

Si hablamos de Romero Brest es sabido que fue el creador de la denominación Educación Física. Estudió Medicina y su título de tesis fue: "Los ejercicios físicos en la escuela (del punto de vista higiénico)". Ya con el nombre de su tesis podemos ver cómo se distinguía su carácter pedagógico dentro del Sistema Educativo. Asimismo, dictaba clases de "ejercicios físicos y militares", y creaba años más tarde el Sistema Argentino de Educación Física que fue adoptado para la formación de los docentes de la materia. Si bien Romero Brest analizó la gimnasia, los juegos y los deportes en sus investigaciones en esta pesquisa nos enfocaremos en la gimnasia únicamente.

En 1898 comenzó a dictar clases de Educación Física y, se decretó la reorganización completa de la Educación Física secundaria suprimiendo los ejercicios militares por ejercicios físicos oficialmente. Y, en el año, 1901 comenzó a dictar cursos de Educación Física para formar docentes.

Cuando se implementaron los "Ejercicios Físicos" en las escuelas solicitó la construcción del trapecio y las anillas; aunque continuó con el trabajo de barras, manubrios, palenques y cuerdas. Por otro lado, dictó la materia denominada: "Gimnasia teórica aplicada a la mecánica humana, Ventajas de los pequeños aparatos sobre los grandes aparatos, Anatomía, Fisiología e Higiene, Pedagogía, e Historia de la gimnasia y esgrima". Romero Brest le daba mucha importancia a la gimnasia, aunque entendía al cuerpo como una construcción biológica con músculos, articulaciones, huesos y sistemas: "el ejercicio físico en esta edad no sólo es un sedante poderoso de la excitabilidad nerviosa, cuya indicación llena por completo, sino que también contribuye de una manera notable y cierta al desarrollo, crecimiento y perfeccionamiento armónico de todo el organismo...”. (Romero Brest, 1900, p.28)

Asimismo, se ocupó de enseñar a los varones ejercicios de velocidad, de fuerza como los saltos y trabajos en el tronco, de equilibrio, de resistencia con prácticas sofocantes y respiratorias, de locomoción, ejercicios gimnásticos sin aparatos como las suspensiones, rondas escolares, excursiones, deportes y juegos. Cada uno de ellos con sus respectivas indicaciones, contraindicaciones, graduaciones, intensidades y una clasificación pertinente de los mismos con soporte higiénico y científico. También se preocupó por los momentos de descanso de los alumnos, la vestimenta y la alimentación.

Para las mujeres había ejercicios de correr, saltar, ejercicios para prepararlas para la maternidad, ejercicios estéticos, armonía de las formas, la gracia de movimientos, ejercicios suaves y elegantes manteniendo el pudor y el rescato, los ejercicios de fuerza debían ser delicados, los ejercicios de velocidad debían ser limitados, etc. Para los hombres, los ejercicios eran relacionados a la lucha, las carreras, las cinchadas, saltos, trabajos de fuerza, velocidad y resistencia, la ponderación del coraje, la osadía, y el carácter fuerte y enérgico, etc. Todos ejercicios que afianzaban la virilidad y dejaban de lado la femineidad.

Si bien, los ejercicios físicos podrían proponer una gran diferencia de género, no se le puede negar a Romero Brest la importancia de sus aportes a la Educación Física, su compromiso y el carácter pedagógico que impulsó en las escuelas dejando de lado la gimnasia militar.

\section{Tercer momento: LA gimnasia atraVESADA Y UTILIZADA POR LOS PARADIGMAS DE LA EdUCACIÓN Física. El PARAdigma PSICOMOTRIZ Y EL PARADIGMA FísICO DEPORTIVO}

Como ya se expresó, la gimnasia ha pasado por varios procesos de encuentros y desencuentros. El paradigma psicomotriz y el físico deportivo son dos de los que más atravesaron nuestras prácticas. Dos paradigmas más contemporáneos que utilizaron a la gimnasia como escudo para entrenar a quienes la practicaban.

El paradigma físico-deportivo transcurre en el siglo XVIII con un fuerte vínculo con la ciencia positiva y sus discursos científicos constituidos como criterios de verdad. 
El modelo de rendimiento del mismo se orientó hacia lo técnico del deporte de elite; utilizando métodos analíticos y conductistas que convirtieron a la Educación Física en una disciplina meramente técnica a través de la práctica (de lo simple a lo complejo), el error y la demostración (estímulo-respuesta). De este modo, el resultado era la búsqueda del rendimiento, la competición y la demostración.

Asimismo, para medir capacidades y habilidades aparecían los test de evaluación que permitían un control rápido y riguroso de la educación (se buscaban resultados concretos en todos los test disponiéndolos en una media standart estadística para saber quiénes alcanzaban o no los objetivos).

Con este modelo, por un lado, la gimnasia aparecía como un puente necesario para entrenar el cuerpo y corregir todo tipo de lesiones, malas posturas o malos hábitos a través del esfuerzo, el sacrificio, la fuerza de voluntad, el autocontrol y la disciplina. Por otro lado, sirvió para el entrenamiento de las capacidades motoras y condicionales con el objetivo optimizar el rendimiento de los deportistas. Y también, en la toma de los test de evaluación de cada una de las capacidades condicionales (velocidad, resistencia, fuerza y flexibilidad) y coordinativas (equilibrio, coordinación, acomplamiento, etc.).

Por otro lado, Educación Psicomotriz aparecía de la mano de Dupré y Le Boulch en nuestro país en 1913. Fue atravesada por la Gestalt, la fenomenología, el pensamiento dialéctico y la epistemología genética.

Según este paradigma las experiencias vividas por cada individuo se transfieren luego en las diferentes áreas educativas. Por ello trabaja sobre lo consciente construyendo una representación psíquica de su cuerpo y de sus posibilidades. El cuerpo, en este sentido, es entendido como un organismo de totalidad y unidad (dualidad) que piensa y siente (psicosomático).

Abordó temas como la rehabilitación, la reeducación motriz y el trabajo con trastornos mentales y neuromotrices; aunque se buscaba prevenir más que curar.

Desde la gimnasia únicamente se trabajaba el equilibrio, la armonía, la simetría, el espacio, los objetos, el esquema corporal, la percepción temporal, la lectura, la escritura, las habilidades motoras, el domino de los movimientos y el juego. Los docentes de Educación Física muchas veces sólo eran auxiliares de los profesores de lengua y literatura porque a través de la escritura, la caligrafía y la lectura correcta se suponía que el alumno podía llegar a transferir las experiencias vividas mentalmente hacia su cuerpo.

Ninguno de estos paradigmas toma a la gimnasia como contenido. Por el contrario, la utilizan en su beneficio y la fragmentan quedando relegada a una mera entrada en calor o acondicionamiento físico, o la práctica de actividades aisladas.

Ambos piensan en un cuerpo orgánico y psicosomático. Un cuerpo perfectamente entrenable que pueda dar respuestas rápidas, en cada uno de los deportes, a aquellos problemas que se presentan en la práctica. Tomando al sujeto como individuo a estudiar y analizar poniendo a la gimnasia como un recurso y no como una práctica con un valor educativo, sistemático e intencional.

\section{Cuarto momento: La gimnasia de los Diseños Curriculares en el Período de 2007} AL 2017

Ya desde el año 1999 la mirada de los Diseños curriculares partía desde el sujeto que aprende. Años más tarde se retomaron estas ideas y se profundizó en el fortalecimiento y enriquecimiento de la Educación Física. Se pensó en una práctica pedagógica, humanista y social que, dejaba de lado el conductismo, cuyos objetivos fueron: 1) favorecer la formación integral, 2) favorecer la formación grupal y política, y 3) poner énfasis en la formación para el mundo del trabajo. Asimismo, la autonomía personal, la sensibilidad corporal propia y de los otros, el reconocimiento de ser sujeto de derecho, y la disponibilidad hábil de una motricidad vinculante, creadora y productiva, eran las líneas generales.

En este Diseño Curricular del 2007 se plantearon dos conceptos claves: la corporeidad y la motricidad. La corporeidad entendida como la presencia al mundo (el cuerpo) y lo que permite captar la realidad en sus diferentes dimensiones desde las emociones, los pensamientos, la comunicación y la sensibilidad. Desde esta 
perspectiva hay dos líneas concretas de pensamiento. Gruppé entiende que: "el hombre vive como cuerpo, se experimenta así mismo como cuerpo y vive su cuerpo como suyo. Conoce su cuerpo, toma posturas frente a él. Puede distanciarse de él: puede disponer su corporeidad" (Gruppé, 1976, p.42). Asimismo, hay dos autores que continúan con el análisis de este concepto: "la persona se manifiesta a través y con su cuerpo pero estas manifestaciones - sentimientos, pensamientos- son parte de ese cuerpo. Hablar de cuerpo en toda su amplitud es trascendente del sistema orgánico, para entender y comprender al propio humanes. El humanes ya no sólo posee un cuerpo (que sólo hace) sino que su existencia es corporeidad, y la corporeidad de la existencia es corporeidad, y la corporeidad de la existencia humana implica hacer, saber, pensar, sentir, comunicar y querer" (Rey Cao y Trigo Aza, 2001, p.3). Por otro lado, como manifestación de la corporeidad, la motricidad, posibilita a los sujetos vincularse con otros, conocer el mundo y conocerse a sí mismo. A partir de las diferentes experiencias que le permitan tanto apropiarse de la cultura como producirla.

Ahora bien, sobre estos conceptos que se han desarrollado en el anteúltimo Diseño Curricular de la Provincia de Buenos Aires, habría que revisar qué rol cumplieron y cómo atravesaron a la gimnasia en la currícula educativa: ¿fue una gimnasia que mejoró los movimientos conscientes e inconscientes?, ¿mejoró la relación de los alumnos con su propio cuerpo y con el de los demás?, ¿tuvo un rol protagónico?, ¿fue una gimnasia de ejercicios?, ¿una gimnasia mecánica?, ¿una gimnasia crítica?, ¿una gimnasia que promueve la planificación del interés de los alumnos?, ¿una gimnasia que propone un aprendizaje en conjunto?, ¿una gimnasia que se entrena o que se reflexiona?, ¿una gimnasia con criterios de evaluación claros y precisos que parten desde el sujeto que aprende?

En esta revisión del Diseño había un espacio definido para la gimnasia: el eje corporeidad y motricidad. Si bien, en el enunciado de los ejes no se habla de gimnasia queda en evidencia el rol que tuvo por los contenidos que se especifican en el eje. Por ejemplo, en el segundo ciclo de la Educación Primaria, las expectativas de logro mencionaban: usar habilidades motoras con ajuste coordinativo, en situaciones gimnásticas, de juegos sociomotor y deportivo en distintos ámbitos, construir actitudes y posturas corporales a partir de una mayor sensibilización y conciencia corporal, identificar las capacidades condicionales y su relación con el accionar motor, inventar formas de expresión y comunicación corporal y gestual en actividades sociomotrices, gimnásticas y expresivas. Dentro de estas expectativas, los contenidos que se corresponden son: las capacidades motoras (resistencia aeróbica, fuerza rápida, flexibilidad y velocidad), las capacidades coordinativas (acople, equilibrio, diferenciación, fluidez, orientación y ubicación espacial y ajuste rítmico), la conciencia corporal (actitudes, posturas, sensaciones de placer y esfuerzo, predominancia lateral) y las habilidades motoras (de desplazamiento, dominio corporal y manipulación). Algo similar ocurre con los contenidos del Primer Ciclo.

En relación a los años anteriores, la gimnasia empezó a tener un lugar importante; toma otro rol en la educación intentando dejar de ser un contenido entrenable para empezar a ser reflexivo. Intentando dejar de pensar en los niños y niñas como objetos de aprendizaje, para pasar a ser sujetos de derechos que construyen su propio saber; aunque lamentablemente pasó a ser un contenido poco enseñable y únicamente de niñas. En este sentido, habría que analizar si en las planificaciones docentes la gimnasia tuvo este carácter reflexivo. Algunos de los que continuaron enseñando este contenido continuaron con la misma metodología tecnicista y directiva, otros la utilizaron únicamente para la primera parte de la clase o para entrenar a los alumnos para el deporte que se iba a enseñar, y otros ni siquiera daban gimnasia. Asimismo, la gimnasia fue desapareciendo como tal de las planificaciones anuales de los docentes quizás por no saber cómo enseñarla de una forma diferente, por falta de capacitación, por desinterés, por darle importancia más a otros contenidos de la Educación Física, etc.; apareciendo relegada a los deportes y los juegos tanto para el primer ciclo como para el segundo ciclo. 
Quinto MOMENTO: LA NUEVA CuRRicula EDUCATIVA EN EL AÑo 2018 y EL ROL DE LA gimnasia. El cambio de la propuesta de Educación Física del Diseño CurRicular de la Provincia de Buenos Aires

Con el nuevo Diseño Curricular la enseñanza de la gimnasia ha ido cambiando el enfoque. Continúan valorizando los conceptos de Corporeidad y Motricidad como en la anterior perspectiva; los (re)afirma pero a la vez, le da un concepto más acabado. En este sentido, entiende la corporeidad como "la presencia en el mundo de los sujetos, constituye una dimensión significativa del ser humano que se va construyendo en el transcurso de la vida. Esta construcción es simultáneamente orgánica y subjetiva y se produce en un desarrollo donde ambos aspectos se influyen en forma mutua y constante" (Diseño Curricular de la Provincia de Buenos Aires, 2018, p.372). Y, por otra parte, la motricidad la configura como la "acción intencionada de la corporeidad, permite a los niños participar, recrear y concretar propuestas referidas a la cultura corporal para conocer y situarse activamente en los contextos donde se desempeñan y desempeñarán" (Diseño Curricular de la Provincia de Buenos Aires, 2018, p.372).

En esta nueva perspectiva, se dejó de lado la expresión "expectativas de logro" y aparecen dos claros enunciados: los propósitos del área y del ciclo, y los objetivos de la unidad pedagógica. Por ejemplo, para el Primer Ciclo de la Primaria se busca: "favorecer la apropiación y utilización de habilidades motores básicas y combinadas, con objetos y sin ellos, en diferentes situaciones motrices con utilización de nociones corporales, espaciales y temporales", "facilitar la exploración y el reconocimiento de las capacidades motoras en las acciones motrices que se pongan en juego en las diversas prácticas corporales", y "promover la elaboración de formas de expresión y comunicación corporal y gestual de modo individual y grupal, integrando diferentes acciones, posiciones y posturas" (Diseño Curricular de la Provincia de Buenos Aires, 2018, p.375). Y, en cuanto a los objetivos, los que hacen referencia a la gimnasia son: "utilizar habilidades básicas y combinadas en diversas situaciones con incremento paulatino de la seguridad y el control", "reconocer la presencia combinada de nociones espaciales y/o temporales en las acciones motoras", "explorar y reconocer las capacidades condicionales en sus acciones motrices", "manifestar formas de expresión y comunicación corporal y gestual en forma individual y grupal” (Diseño Curricular de la Provincia de Buenos Aires, 2018).

En este sentido, los contenidos del Primer Ciclo de la Primaria que se relacionan con la gimnasia bajo el bloque Conciencia y Constitución corporal son: reconocimiento de las posibilidades motrices globales, identificación y utilización de nociones sobre su propio cuerpo, utilización de la predominancia corporal, reconocimiento de la derecha y la izquierda en su propio cuerpo, reconocimiento de la fuerza y la velocidad en algunas acciones motrices, entre otros. Por otro lado, bajo el bloque "Prácticas gimnásticas, atléticas y acuáticas", los contenidos que se relacionan con la gimnasia son: nociones espaciales y temporales en las acciones motoras, exploración de diferentes formas de equilibrio y reequilibración, variando apoyos y alturas, experimentación de trepas, suspensiones y balanceos en aparatos y objetos diversos, apoyos en equilibrio sobre manos y combinando cantidad y tipos de contactos, rolidos de diferentes ejes corporales con control global de movimiento, control del equilibrio en los giros en uno y otro sentido, y acciones de flexión y extensión del cuerpo y giros en forma aislada y en combinaciones simples.

Una modalidad de esta nueva propuesta distinta es incluir a la gimnasia artística y deportiva. ${ }^{1}$ Por ejemplo, los contenidos para el Sexto Año de la Educación Primaria son: vertical a una pierna y vertical balanza con control parcial del equilibrio, rol adelante y atrás con diferentes entradas y salidas, iniciación a acciones que integren posiciones, posturas, equilibrio y apoyo con uno o dos compañeros. Esta gimnasia, si bien no estaba especificada en la propuesta de Educación Física anterior, se había dejado de dictar en las escuelas por falta de presupuesto, por falta de materiales (colchonetas adecuadas, anillas, bigas, barras paralelas, potro, etc.), por falta de interés en los docentes (desconocimiento de su enseñanza o por priorizar otros contenidos de la Educación Física) y también por problemas con su enseñanza y el cuerpo de los menores. En los últimos años se han realizado un cúmulo de denuncias a docentes de Educación Física por abuso y eso ha mermado 
el deseo de los mismos de enseñar gimnasia artística por el contacto corporal que, algunas veces, es necesario para el pasaje del cuerpo.

Los contenidos en esta perspectiva son más amplios y no tan específicos como en la propuesta del 2007. Habría que ver si, finalmente, los docentes empezaron a poner a la gimnasia en sus planificaciones o si continúan relegándola y, dándole prioridad a los juegos y deportes.

\section{CONCEPTUALIZAR A LA GIMNASIA: REVALORIZANDO LAS PRÁCTICAS EDUCATIVAS}

La gimnasia entendida como práctica política, cultural, social e histórica permite mejorar la relación del sujeto con su cuerpo, con los movimientos conscientes e inconscientes, con su cultura y con los otros a través de los diferentes movimientos y técnicas que fueron configuradas, pero que también continúan configurándose con el paso del tiempo.

Esta práctica tiene carácter educativo, intencional y sistemático; aunque también está atravesada por la estética y el consumo. El culto a la belleza y los parámetros sociales con respecto a las exigencias de conseguir un cuerpo hermoso y delgado atravesaron los discursos de lo normal y lo aceptable, también, en el ámbito escolar en niños y niñas de la Educación Primaria.

A lo largo de los años, varias pesquisas se dedicaron a estudiarla, analizarla e indagarla, aunque hoy en día tenga un rol secundario en las planificaciones de muchos docentes de Educación Física. Contenidos como las prácticas deportivas y los juego sociomotores y deportivos parecen multiplicarse a la hora de proyectar el ciclo lectivo en el nivel primario.

Algunas cuestiones que se podrían pensar que la gimnasia no tiene el mismo interés que los demás contenidos de la Educación Física son: a) es aburrida, b) el docente no encuentra la forma de captar positivamente la atención de los alumnos, c) sirve para acondicionamiento del deporte, d) los docentes no tienen las suficientes herramientas para dictar gimnasia durante un período prolongado o una unidad didáctica, temática o proyecto, o no sienten seguridad para realizar una planificación que pondere a la gimnasia en un lugar importante (el mismo lugar importante que tienen los deportes y los juegos), e) se evalúa por medio de mediciones, $\mathrm{f}$ ) las experiencias personales de los docentes no han estado tan vinculadas con la práctica de este contenidos, g) los planes de estudio no le dan la misma importancia que la que se le da a los deportes, h) las políticas estatales desde hace años privilegian al deporte (configuración de la Secretaria de Deportes, Juegos Bonaerenses, Juegos de la juventud, capacitaciones oficiales en deportes, becas a deportistas, etc.), etc.

Ahora bien si la Educación Física y, específicamente la gimnasia, como área pedagógica tiene como objeto de estudio intervenir en la constitución corporal y motriz de los niñas y niños -colaborando en su formación integral y en la apropiación de bienes culturales como este Diseño Curricular de la Provincia de Buenos Aires- surgen las siguientes preguntas: ¿̨por qué la gimnasia se ha dejado de practicar en las clases de nuestra área?, ¿será, quizás, porque las prácticas iniciales de la gimnasia han tenido un recorrido militar y los docentes quisieron alejarse de ello?, ¿será que en el Diseño Curricular anterior la Educación Física físico-deportiva tuvo un tanta preponderancia que la gimnasia quedó relegada?, etc.

Con este panorama poco alentador la gimnasia en las escuelas sigue desapareciendo. Los docentes prácticamente no la dictan, ni la proyectan. La gimnasia progresivamente pierde su valor y su espacio quitándole a los alumnos la posibilidad de: 1) configurar movimientos, 2) aprender modos correctos para sentarse, caminar, correr, agacharse, acostarse, 3) comprender el sentido musical y sus tiempos para poner al cuerpo en movimiento, 4) entrenar capacidades adecuadamente y conscientemente, 5) mejorar su disponibilidad corporal, etc.

En las investigaciones de Mariano Giraldes, reconocido profesor en el área de la gimnasia, propuso utilizar de cada una de las corrientes de la gimnasia aquello que sea apropiado para el grupo, para los objetivos del docente, para su planificación, etc. Cada de una de estas corrientes pueden ser enmarcadas en distintos tipos 
de gimnasias. Por un lado, las gimnasias orientales como el yoga, el Tai chi kung, la danza china, etc. Asimismo, las gimnasias formativas como la alemana. Las gimnasias, deportivas como la gimnasia artística y deportiva, la gimnasia rítmica, la aeróbica de competición y el Crossfit. También están las gimnasias blandas como la eutonía, la senso-percepción, la anti-gimnasia, Feldenkrais, el método Alexander, la gimnasia correctiva, Pilates, etc. Las gimnasias duras como la danesa, la sueca, el método natural de George Heber, moderna, gimnasia natural austriaca, etc. Y, por último, las gimnasias fitness como la aeróbica, el step, la localizada, las de las empresas Radical Fitness, Body systems, Zumba, etc.

Cada una de estas técnicas son de distintas procedencias y pueden hacer un gran aporte a la calidad educativa. Desde esta perspectiva y, avanzando con la importancia de la práctica de la gimnasia y con la necesidad de revalorizarla, es necesario comprender que para enseñarla es preciso tener en cuenta los tiempos de los aprendizajes de los alumnos, sus experiencias previas con lo corporal, el contexto, la invención de un modo de resolver los problemas que para ese momento sea oportuno para los alumnos, etc.

El docente debería enseñar una batería de técnicas que puedan servirle al alumno como herramientas para luego poder construir sus propias prácticas mejorando su relación con el cuerpo e intentando ampliar el repertorio motriz de los alumnos. El dominio de la técnica es importante en la medida que el alumno aprenda a dominar su cuerpo y mejore su disponibilidad corporal en diversas situaciones motrices para adquirir más habilidades, sin buscar resultados físicos; como antes se esperaba de la gimnasia. Por ello, el contenido a enseñar debería ser abierto y múltiple.

La gimnasia en el Nivel Primario debería aparecer como guía de aprendizaje y no como el portador de la verdad del saber; favoreciendo el intercambio entre los alumnos y el conocimiento a partir del uso inteligente del cuerpo y los aprendizajes.

También, sería importante que se seleccionen los criterios de valoración para favorecer dicho aprendizaje, y que las actividades produzcan resultados diversos y divergentes. Según Portos: "la actividad debería llevar a la aceptación consciente del riesgo, la posibilidad del fracaso, de la utilidad de la crítica y la existencia de la incertidumbre que obliga a salirse de caminos transitados, ya aprobados. Esto supone decir que el error, la equivocación, forman parte de este proceso de aprendizaje. El hecho de no cumplir las expectativas no implica la desaprobación, sino la posibilidad de mejorar los aspectos insuficientes para una próxima vez" (Portos, 2011, p.4).

Al finaliza la clase les preguntará: ¿qué sintieron?, ¿cómo lo hicieron?, ¿qué tema se había trabajado?, ¿para qué sirvió?, ¿por qué se hizo de tal forma, se podría haber hecho de otra? ¿Por ejemplo, cómo?, ¿se puede mejorar?, ¿qué estuvo bien?, ¿qué faltó?, ¿cómo resultó el trabajo en equipo?, ¿y el trabajo individual?, etc.

Los alumnos en la medida que aprenden técnicas, que mejoran su relación con el cuerpo, con sus movimientos y los demás, se plantean y resuelven problemas, toman decisiones, prueban, inventan, exploran, observan, escuchan, imitan, copian, aprenden a entrenar, imaginan, comparan, clasifican, evalúan, reflexionan, examinan, se detienen a analizar, etc. Esto permite que, a través de la gimnasia, se favorece la aplicación y el uso inteligente de los aprendizajes que atraviesan lo corporal y mejoran la disponibilidad de los alumnos.

\section{A MODO DE CIERRE PROVISORIO}

Cada uno de estos momentos de la gimnasia ponen de manifiesto los grandes cambios que hubo en esta práctica. Desde la gimnasia militarizada hasta nuestros días podemos dar cuenta cómo se ha ido pedagogizando y cómo a modificando su objeto de estudio.

En el inicio el objeto de estudio estaba centrado en el hombre fuerte y ágil al que había que preparar para la guerra, porque los niños y niñas eran entendidos como hombres en miniatura. Décadas más tarde, con un cambio de paradigma se deja de entrenar a estos hombres en miniatura, y se les da el lugar que se merecen. Se los reconoce como niños y niñas con derechos, aunque las pesquisas estaban centradas en el movimiento y 
el hacer; a la vez que había una marcada diferencia de género al practicas Educación Física. Si bien no era un entrenamiento estricto las bases fisiológicas estaban tenidas en cuenta. Con los paradigmas físico-deportivo y psicomotriz el objeto de estudio estaba centrado en el conductismo para lograr un buen entrenamiento, y el aparato psicomotor, las transferencias y la rehabilitación de las personas. La gimnasia servía de auxilio de los otros contenidos o áreas aparentemente más importantes. Años más tarde, en el 2007, el enfoque de la gimnasia volvió a cambiar. Las investigaciones comenzaron a centrarse en el sujeto que aprende, aunque también la mirada estaba en el hacer. Por último, en la actualidad, finalmente en objeto de estudio continúa siendo en el sujeto que aprende, pero la mirada parecería que se centra en el ser. La gimnasia tiene un lugar mucho más presente a través de las capacidades coordinativas y condicionales, pero también se pone de manifiesto la enseñanza de la gimnasia artística y deportiva.

Tantos paradigmas y tantas corrientes de la gimnasia la atravesaron desde sus inicios que merece tener un lugar importante en la curricula del área de Educación Física, donde los profesores puedan (re)valorarla y ponerla en práctica a través de sus planificaciones anuales. Planificaciones que tengan en cuenta a los deportes, los juegos, la natación, la vida en la naturaleza (o el medio ambiente como propone la propuesta de Educación Física del Diseño Curricular) y la gimnasia. Todos los contenidos en la misma medida y con la misma importancia.

La gimnasia debería dejar de ser meramente un conjunto de técnicas a reproducir e imitar. Es necesario considerarla como un conjunto de técnicas, pero carácter educativo y pedagógico, que favorezca el uso inteligente propio del cuerpo, con los demás, con los movimientos, con los objetos y que está inmerso en una cultura específica pero que es capaz de aprender de otras para mejorar su relación. Donde los alumnos son los protagonistas de su aprendizaje en la construcción de modos de moverse, de modos de ser, sentir, pensar, reflexionar, analizar, seleccionar, evaluar, etc.

En este sentido, se propone, por último, enseñar las diferentes corrientes de las gimnasias antiguas y nuevas gimnasia a través de un uso criterioso, inteligente, diverso y reflexivo para revalorizar la gimnasia, pero cambiando la forma de enseñar.

\section{REFERENCIAS}

Agüero L., Iglesias S. y Del Valle Milanino, A. (2009). Enrique Romero Brest y los inicios de la educación física escolar. Su tiempo, su vida, su pensamiento y su obra. Revista eä, 1(1), 1-38. Recuperado de http://www.ea-journal.co $\mathrm{m} / \mathrm{art} /$ Enrique-Romero-Brest.pdf

Aisenstein, A. (2006). Cuerpo, escuela y pedagogía. Argentina, siglos XIX y XX. En A. Aisenstein y P. Schragrodsky, Tras las huellas de la Educación Física escolar argentina. Cuerpo, género y pedagogía 1880-1950 (pp. 19-42). Buenos Aires: Prometeo Libros.

Bulus, V. (2011). Los problemas de las gimnasias. Educación Física y Ciencia, 13, 149-155. Recuperado de http://ww w.redalyc.org/pdf/4399/439942655011.pdf

Carballo, C. (1999). Educación Física: Crisis epistemológica, confusión didáctica y alternativas de investigación. Trabajo presentado en Primer Congreso Nacional en Investigación Educativa. Universidad Nacional de Comahue, Cipoletti.

Diseño Curricular de la Provincia de Buenos Aires. Propuesta de Educación Física del año 2007.

Dirección General de Cultura y Educación de la Provincia de Buenos Aires (2018). Diseño curricular para la educación primaria: primer ciclo y segundo ciclo. Recuperado de http://servicios.abc.gov.ar/lainstitucion/organismos/cons ejogeneral/disenioscurriculares/primaria/2018/dis-curricular-PBA-completo.pdf

Fridman, C. (1999). La Educación psicomotriz. Una falacia de nuestra época. Trabajo presentado en el 1er Congreso Nacional de Investigación Educativa. Universidad Nacional de Comahué. Cipoletti.

Galak, E. y Napolitano, E. (2009). Discursos sobre el cuerpo en las investigaciones en Educación Física. Trabajo presentado en el 8vo Congreso Argentino y 3ero Latinoamericano de Educación Física y Ciencias. Universidad Nacional 
de La Plata, Buenos Aires. Recuperado de http://www.memoria.fahce.unlp.edu.ar/trab_eventos/ev.7967/ev.7 967.pdf

Giraldes, M. (2001). Gimnasia el futuro anterior. De rechazos, retornos y renovaciones. Buenos Aires: Editorial Stadium. Gruppé, O. (1976). Teoría pedagógica de la Educación Física. Madrid: INEF.

Mamonde, M. (1995). Educación Física militarizada en Argentina. Educación Física y Ciencia, 1, 46-51. Recuperado de http://www.memoria.fahce.unlp.edu.ar/art_revistas/pr.258/pr.258.pdf.

Portos, M. E. (2011). ¿Qué enseñamos cuando enseñamos Gimnasia Artística?. Trabajo presentado en IX Congreso Argentino y IV Latinoamericano de Educación Física y Ciencias. Universidad Nacional de La Plata, Buenos Aires. Recuperado de http://www.memoria.fahce.unlp.edu.ar/library? $\mathrm{a}=\mathrm{d} \& \mathrm{c}=$ eventos\&d=Jev 9817

Rey Cao, A. y Trigo Aza, E. (2001). Motricidad ¿Quién eres? Apunts. Educación Física y Ciencia, 59, 91-98.

Schragrodsky, P. (2001). Juntos, pero no revueltos: la Educación Física mixta en clave de género. Nomadas, (14), 142-154.

Scharagrodsky, P. (2004). El padre de la Educación Física Argentina: fabricando una política corporal generalizada (1900-1940). Revista Perspectiva, Revista do Centro de Ciencias da Educaçao, 22, 83-119.

Scharagrodsky, P. (2006). Los ejercicios militares en la escuela argentina: modelando cuerpos masculinos y patriotas a fines del siglo XIX. En A. Aisenstein y P. Scharagrodsky, Tras las huellas de la Educación Física escolar argentina. Cuerpo, género y pedagogía 1880-1950. Buenos Aires: Prometeo.

\section{Notas}

1 Cabe destacar que la gimnasia artística y deportiva no está dentro de las corrientes de la gimnasia, ya que es un deporte reglado, institucionalizado y competitivo. 